\title{
LA MORPHOSYNTAXE DU FRANÇAIS TCHATÉ : LE CAS DU SYNTAGME VERBAL CONJUGUÉ
}

\author{
Jan LAZAR \\ Université d'Ostrava \\ Université d'Opole
}

\begin{abstract}
En): We live in a period which is inseparably linked to new forms of electronic communication. The Internet provides its users with new forms of communication including messaging, discussions, and chats. It appears that the French language used in email messages, forums, blogs and chat sessions differs from the standard written language. The computer has become a tool, a sort of mediator, which indirectly modifies the CMC discourse. The new 'genre' is commonly referred to as "computer-mediated communication" (CMC) in English linguistics, whereas French linguistics uses different terms including: "oral-written hybrid forms" (ANIS, 1998, 1999, 2001), "Cyberl@ngue" (DEJOND, MERCIER, 2002) or mediated electronic discourse (PANCKHURST, 1998, 1999). The aim of our contribution is to analyze specific forms of such type of written communication which appear in French chats, and their influence on the morphological structure of subject-verb agreement. It should be noted that online communication takes place in real time because the message appears immediately after "Enter" is pressed, which means that chat discussions have a conversational character (ANIS, 1999 : 71). In order not to lose the progression of conversation with other chatters, it is necessary to react as quickly as possible, because the message remains very little time on the screen. As a result, the users abbreviate the messages so as to keep up with the chat communication. According to JANDOVÁ $(2006,22)$ the messages on chats are generally composed of six words. This economy of expression necessarily leads to a reduction of morphological structure of the discourse.
\end{abstract}

Keywords (En): Internet, chat, written communication, subject-verb agreement.

Mots-clés (Fr): Internet; chat; communication écrite; accord sujet-verbe.

\section{Introduction}

GREVISSE (1991 : 686) définit l'accord comme « le fait qu'un mot variable (que nous appelons receveur) reçoit d'un autre mot de la phrase (mot que nous appelons donneur) ses particularités morphologiques : son genre, son nombre et sa personne. » En même temps, il nous précise que l'accord est un phénomène qui se marque plus dans l'écrit que dans l'oral (GREVISSE, ibidem). GUIRAUD (1974 : 64) va plus loin en constatant que « $C$ 'est un des traits fondamentaux du français que l'accord n'y est qu'une survivance maintenue par l'action arbitraire des grammairiens. Il survit et sévit surtout dans l'orthographe et n'existe qu'à l'état de vestige dans la langue parlée. » À titre d'exemple, on peut reprendre une de ses phrases modèles et sa transcription phonétique :

\section{Les (PL1) filles (PL2) regardent (PL3) la télévision - [1e (PL1) fij regard la televizjõ]}

Cette phrase met en évidence que la langue française marque le nombre dans le code graphique d'une façon hautement redondante en indiquant le pluriel par le $s$ sur le déterminant (PL1) et le sujet (PL2) et, en plus, en modifiant la désinence verbale (PL3). HONOVÁ (2008 : 99) nous précise qu'en comparaison avec les langues slaves, le système temporel du français est beaucoup plus riche et se 
manifeste par des marques morphologiques plus variées qu'en tchèque. Par contre, le code phonique ne marque le pluriel qu'une seule fois (PL1), par la présence du [e] sur le déterminant. Il va de soi que le français standard n'autorise pas la suppression des marques morphologiques du nombre et du genre, quoiqu'elles ne se réalisent pas dans le code phonique. Pourtant, la situation se révèle différente dans la communication médiée par ordinateur, notamment en ce qui concerne le français tchaté. Reprenons quelques exemples tirés de notre corpus du français tchaté :

\author{
looveur_42 > de se ke tu veu \\ sebas_tbol > lililatigresse > si tu vien a lafin de cette anné, ca sera agreable \\ lililatigresse > ricardo30 > oui oui on dis ça \\ caro74 > se pa..leque?
}

Discuteur31303 > j'habit dans le maroc et toi

Les exemples, mentionnés supra, nous montrent clairement que la morphosyntaxe du français tchaté diffère considérablement du français écrit standard. Les premières études consacrées au phénomène du français tchaté (PIEROZAK, 2000 a, b, 2003 a, b, 2005 ; ChOVANCOVÁ, 2008 a, b) n'abordent les questions morphosyntaxiques que d'une manière marginale en s'intéressant notamment aux pratiques scripturales particulières et à leur menace potentielle pour la langue française soutenue. Nous jugeons nécessaire de combler cette lacune en consacrant cet article à un problème morphosyntaxique particulier, c'està-dire le cas de l'accord sujet pronominal et le verbe conjugué.

\title{
Quelques caractéristiques de la communication tchatée
}

Rappelons que la communication médiée par ordinateur peut être caractérisée du point de vue de la synchronicité qui influence le caractère de l'interaction entre les internautes (CHOVANCOVÁ, 2008a: 12). Étant donné que la communication tchatée exige que l'émetteur et le récepteur soient connectés au même moment, on peut attribuer le caractère de synchronicité à ce type de communication. Il faut préciser que la communication sur un chat se déroule avec une extrême rapidité, si bien que les tchateurs doivent réagir le plus tôt possible, pour ne pas perdre le fil de la conversation. En même temps, il faut ajouter que la communication tchatée s'approche de la communication orale spontanée (JANDOVÁ, $2006: 88$ ) et juge les marques morphologiques qui ne sont pas prononcées à l'oral comme redondantes. Il en résulte que l'économie de l'expression reste une des caractéristiques essentielles de cet environnement communicatif (DEJOND ; MERCIER, 2002 : 35). En envoyant un message sur l'écran d'un ordinateur, il faut dire le maximum avec le minimum de caractères, ce qui tend logiquement à la réduction des marques morphologiques. L'objectif de ces réductions est de faire correspondre le code graphique et le code oral de manière qu'à chaque graphème corresponde un phonème concret, ce qui rapproche la langue française des langues dont la 
correspondance phonético-graphique est beaucoup plus transparente (KATZ; FROST, 1992). Précisons que PIEROZAK (2005) observe une certaine ressemblance entre le français tchaté et les créoles à base française. On observe que les difficultés syntaxiques, posées par les créoles et le français tchaté impliquent, d'une certaine façon ou d'une autre, la notion polymorphe de marque. Pourtant, elle refuse d'attribuer au français tchaté la notion de langue « simple » ou «simplifiée » (PIEROZAK, 2005: 107), mais on peut plutôt parler d'une langue « re-marquée», qui se caractérise par de nouvelles marques morphologiques.

Étant donné l'étendue limitée de cet article, nous ne pouvons pas décrire en détail toutes les réductions des marques morphologiques en français tchaté, mais nous voulons orienter notre attention vers le cas de l'accord sujet pronominal verbe conjugué et sa réalisation graphique en français tchaté. Nous supposons que l'accord canonique, conservant toutes les marques morphologiques du français standard, sera en minorité par rapport à l'accord introduisant des marques morphologiques simplificatrices. Pour observer les différents aspects de notre hypothèse, nous disposons d'un corpus de 1200 messages téléchargés sur trois tchats francophones: Diskut, ${ }^{1}$ Adoskuat, ${ }^{2}$ Chat. ${ }^{3}$ Étant donné que l'objectif de notre travail est d'observer le langage des jeunes locuteurs natifs, nous avons téléchargé notre corpus dans trois salons de clavardage destinés aux jeunes tchateurs (Diskut - salle «moins de 18 ans », Adoskuat - salle « Tchat entre ados », Chat - salle «Ados »). Nous nous sommes connecté à chacun de ces trois tchats deux fois, à des dates et heures différentes, pour pouvoir obtenir le matériel le plus équilibré possible. Rappelons que l'anonymat est une des caractéristiques essentielles de cet environnement communicatif, ce qui ne nous permet pas d'apprendre quoi que ce soit sur l'éducation des internautes dont les énoncés sont analysés. Même si plusieurs tchateurs nous ont adressé la parole, nous n'avons engagé la conversation avec aucun d'entre eux et nous n'avons envoyé de messages ni public ni privé. Il s'ensuit que le langage que nous avons soumis à l'analyse est représentatif du discours tchaté quotidien.

\section{Analyse des résultats}

L'accord du sujet et du verbe représente un cas classique de relation syntaxique : pour la réalisation de ce type d'accord intersyntagmatique, le sujet se doit de relier deux syntagmes, un nominal et un verbal. En français, comme dans un grand nombre d'autres langues, la règle d'accord en nombre du sujet et du verbe est simple : un sujet au singulier demande un verbe au singulier et un sujet au pluriel, un verbe au pluriel. Néanmoins, il faut préciser que certaines constructions peuvent déroger à cette règle, notamment en ce qui concerne les expressions de la collectivité qui peuvent s'accorder soit au singulier, soit au pluriel (HENDRICH ; RADINA ; TLÁSKAL, 1992 : 570). Cette liberté de choix qui permet une certaine variabilité de l'accord peut aussi causer une certaine confusion auprès des locuteurs natifs. Les recherches menées par LAMBERT et KAIL (2001 :

\footnotetext{
${ }^{1}$ http://www.discut.fr

${ }^{2} \mathrm{http} / / / \mathrm{www} \cdot$ adoskuat.com/zone-chat.html

${ }^{3} \mathrm{http}: / /$ www.chat-fr.org
} 
580) prouvent que les locuteurs natifs traitent plus rapidement les phrases comportant une violation de l'accord nominal que celles comportant une violation de l'accord verbal. Précisons que plus la phrase contient d'indices convergents, plus le nombre d'erreurs et le temps de détection sont importants (LAMBERT; KAIL, 2001 : 581). Il en résulte que la proposition «Les danseuses extraordinaires $v a »$ est moins efficacement traitée que la proposition «Jean vont ». Étant donné que le facteur de prédictibilité intervient considérablement dans le traitement de l'accord, nous avons décidé de focaliser notre attention sur l'accord du syntagme verbal conjugué et sa réalisation en français tchaté, qui se caractérise par l'extrême rapidité d'échanges. L'analyse détaillée de notre corpus a relevé 464 cas de cet accord. $\mathrm{Vu}$ qu'ils se manifestent par des marques morphologiques très variées, qui ne correspondent pas aux règles grammaticales du français soutenu, nous avons décidé de les classer en 5 catégories. Avant de passer à l'analyse détaillée de notre corpus, nous jugeons utile de comparer notre classification avec celle de STARK (2011) qui s'intéressait à la morphosyntaxe dans les SMS suisses francophones. En analysant son corpus, STARK a pu identifier 6 types différents de marquage graphique de l'accord sujet-verbe conjugué : 1. Accord canonique qui correspond à notre catégorie $\mathrm{A} 2$. Accord SMS qui correspond à notre catégorie $\mathrm{B} 3$. Accord partiel : absence du sujet qui correspond à notre catégorie $\mathrm{D}$ 4. Accord partiel par le thème verbal que nous ne distinguons pas dans notre corpus 5. Accord partiel : abréviation qui correspond à notre catégorie $\mathrm{C}$ 6. Non-accord qui manifeste des ressemblances avec notre catégorie $\mathrm{E}$, mais à la différence des SMS, le français tchaté conserve toujours le sujet donneur.

A) Le premier type d'accord observé dans notre corpus est l'accord canonique (STARK, 2011 : 40) qui se caractérise par la présence morphologique du donneur et le marquage explicite et canonique de la personne et du nombre sur le verbe conjugué, c'est-à-dire le receveur :

\author{
Lililatigresse > sebas_tbol > il pleut tjrs chez $v$ s \\ beaucoup aussi, on grandit) \\ LiOu34 > Casper > nn c'est juste que j'aime bien le film Casper:p \\ Radis_Noir > Sur_que_non > Je l'ai toujours su \\ Anonyme2325920 > Je change mon pseudo
}

HommeGENTIL > ricardo30 > Mais non ... moi j'ai appris à me fortifier ... :) (On apprend

Ajoutons qu'on observe que les marques morphologiques sont souvent multipliées lors de la communication tchatée. Il s'agit d'un phénomène typique pour la communication médiée par ordinateur, car une pression prolongée sur la touche d'une certaine lettre permet une multiplication facile de celle-ci. MARCOCCIA (2000) nous explique que cette multiplication permet d'ajouter une valeur expressive, émotive et affective aux énoncés tchatés. Ainsi, les tchateurs Laly et Osmose peuvent «crier » en multipliant les désinences du verbe conjugué. 
Laly > 6 eske kekun parleeeeeee $?$ !

Osmose > ricardo30 > oui merci je paaaaaaaaars!

B) Le deuxième type d'accord observé dans notre corpus se caractérise par une graphie déviante par rapport à la graphie standard. Quoique le pronom sujet clitique soit modifié, il est conservé et le receveur contient des marques explicites de la personne et du nombre. On peut constater que ce type d'accord tient à la formation d'un syntagme agglutiné (CHOVANCOVÁ, 2008a : 97). On observe cette agglutination notamment à la première et à la deuxième personne du singulier, qui se manifestent par des marques morphologiques syncrétiques, ce qui pousse les tchateurs à agglutiner le pronom sujet clitique au verbe pour éviter la confusion.

Cylia_77 > Osmose > tpars en we?

HommeGENTIL > Cylia_77 > JJe suis très fort en ce domaine ... Je suis persuadé que t'es pas une femme..

Miko > Moi c'est Miko t'sais comme les glaces.

Clarvac > Le`Cadavre > XD J'vais encore m'faire Kicker $x$ )

taz04 > Mwyann> desoler jmentrai,nepour etreoperatuer :\$

Néanmoins, il faut préciser qu'on trouve aussi des exemples dans lesquels le pronom clitique n'est pas agglutiné au verbe, même si sa réalisation graphique se montre déviante par rapport à la graphie standard. Nous observons cette tendance à la troisième personne du pluriel, qui se manifeste par des marques morphologiques variées, ce qui entraine que les tchateurs ne ressentent pas le besoin de l'agglutiner. Reprenons quelques exemples tirés de notre corpus :

Mapi > sebas_tbol > fais gaf aux majuscules il vont te virer

Socrate_le-boss > Jenn > il sont jolie?

Il convient de préciser qu'une autre interprétation de cet accord est possible. Étant donné que le sujet donneur est au singulier et le verbe receveur est au pluriel, il pourrait appartenir à la catégorie E, c'est-à-dire l'accord erroné. Néanmoins, c'est la réaction des autres tchateurs qui nous précise qu'il s'agit bien de la troisième personne du pluriel, mais les tchateurs ne jugent pas nécessaire d'ajouter la lettre $s$ au donneur, car la forme du receveur marque clairement qu'il s'agit du pluriel.

Mapi > sebas_tbol > fais gaf aux majuscules il vont te virer

sebas_tbol > Mapi > ils peuvent faire ca ???

Socrate_le-boss > Jenn > jaimré bien joué avec tes pied moi

Jenn $>$ Socrate_le-boss $>$ ha ouais?! 
Socrate_le-boss > Jenn > il sont jolie?

C) Le troisième type d'accord reflète encore plus les tendances oralisantes du discours tchaté. Il se caractérise par la présence du sujet pronom clitique donneur et du verbe receveur, mais le receveur qui est affecté par ces tendances oralisantes ne contient pas le marquage explicite de la personne et du nombre, ce qui permet de constater l'absence de l'accord dans ce cas.

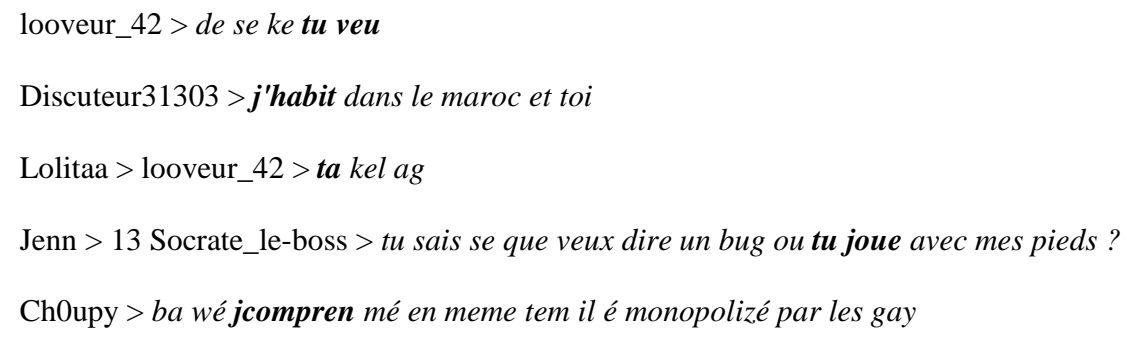

Il nous semble important d'ajouter que les tendances oralisantes du discours tchaté se manifestent aussi par de nouvelles marques morphologiques créatives qui ne sont pas utilisées en français standard. Étant donné qu'elles se caractérisent par la présence du pronom sujet donneur et du verbe receveur, nous jugeons pertinent de les classer dans la même catégorie. On peut constater que les tchateurs se montrent très créatifs en introduisant de nouvelles marques morphologiques dans le système morphosyntaxique de la langue française. Mentionnons quelques exemples :

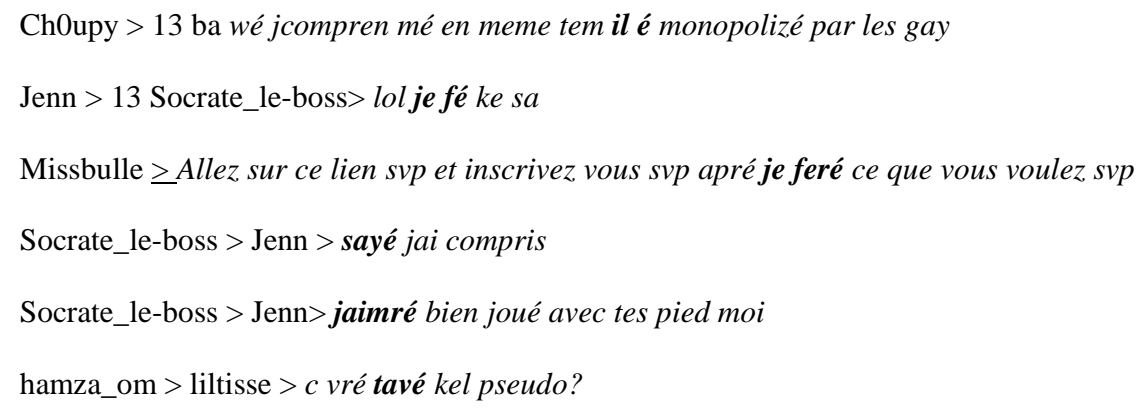

Comme nous le montrent les exemples ci-dessus, le é se révèle la marque morphologique phonétisante la plus répandue dans notre corpus. CHOVANCOVÁ (2008a : 97) attribue à cette marque la dénomination «é-passe partout », car il est susceptible de remplacer les terminaisons du conditionnel et du futur simple ainsi que de l'indicatif et de l'impératif de certains verbes conjugués.

Parmi d'autres marques créatives, nous observons les syllabogrammes qui profitent de la valeur phonétique de certaines lettres de l'alphabet. Ainsi, la lettre $G$ peut facilement remplacer le verbe conjugué avoir - j'ai en réduisant le nombre de caractères de trois à un. 
Liltisse > tnt $\mathbf{g}$ joué avc un hamza

Ch0upy > $13 \mathrm{~g}$ rien contre lé gays mé bon voila koi

Un autre syllabogramme qui apparaît assez fréquemment dans notre corpus est la lettre C. Cette prédilection vient du fait qu'elle peut facilement remplacer les trois personnes du singulier du verbe savoir conjugué au présent de l'indicatif ainsi que la troisième personne du singulier du verbe être conjugué au présent de l'indicatif. Mentionnons quelques exemples tirés de notre corpus :

LiOu34 > Omarion > nn, les pv c pas ma tasse de thé (c'est $\rightarrow \mathrm{c}$ )

Agnam >1Zoe_>c plus un pb de nos jours tu peux avoir des parents lesbienne $c$ pas un soucis (c'est $\rightarrow \mathrm{c}$ )

deesse33 > ouaaaa c plein d'homme (c'est $\rightarrow \mathrm{c})$

sebas_tbol > lililatigresse $>$ c nouveu ce clima a marseille $(\mathrm{c}$ 'est $\rightarrow \mathrm{c}$ )

liltisse > hamza_om > salut hamza. mais on c pas parlé en privé sur ce tchat.c con! (s'est $\rightarrow \mathrm{c})$

ricardo30 > HommeGENTIL > tu c $j$ ai bocoup soufer osi peut etre pa pour la mm chose :) $($ sais $\rightarrow$ c)

ricardo30 > HommeGENTIL > tu c c peut etre un mode de vie de cherché la soufrance $($ sais $\rightarrow$ c, c'est $\rightarrow$ c)

On note aussi la présence de la lettre $\mathrm{T}$ qui se substitue à l'accord du verbe être à la deuxième personne du singulier du présent de l'indicatif.

Socrate_le-boss $>$ Ch0upy $>$ T une fille toi ?? (tu es $\rightarrow$ t'es $\rightarrow \mathrm{T}$ )

BeL_Ange $>$ caro74 $>$ T 1 fille? ( tu es $\rightarrow$ t'es $\rightarrow \mathrm{T}$ )

ehdi90 $>1$ sousou93 $>\mathbf{T}$ très geti! (tu es $\rightarrow$ t'es $\rightarrow$ T)

Ajoutons qu'on trouve aussi la lettre $\mathrm{T}$ qui substitue la troisième personne du singulier du verbe être conjugué à l'imparfait de l'indicatif.

loveur95 > CT impec hier!!!!!! ( c'était $\rightarrow$ T)

Mentionnons aussi la lettre $\mathrm{V}$ qui substitue la première et la deuxième personne du verbe aller conjugué au présent de l'indicatif. Pourtant, sa fréquence est assez limitée par rapport à la lettre C.

deesse33> je $\mathbf{v}$ très bien (vais $\rightarrow \mathbf{v}$ )

D) Le quatrième type d'accord, trouvé dans notre corpus, ne contient pas le donneur et ne conserve que le receveur. L'accord est fait sémantiquement avec le 
pronom omis, considéré comme redondant, puisque le pluriel est donné par le verbe. D'autre part, il est vraisemblable que le fait qu'il s'agisse d'un masculin ou d'un féminin est donné par le contexte.

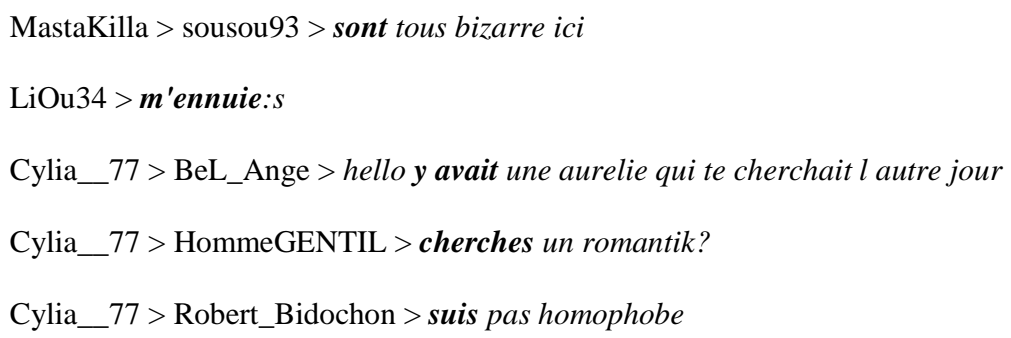

Il nous semble utile de souligner que ce type d'accord affecte le plus souvent le présentatif il y $a$ qui apparaît, dans notre corpus, sous la forme elliptique y $a$ ou $y a$. Précisons qu'il s'agit d'une construction verbale typique pour la communication tchatée, ce que prouvent les recherches menées par VAN COMPERNOLLE ; WILLIAMS (2007 : 61). En analysant trois corpus différents (IRC, Forum, clavardage modéré) ils arrivent à la conclusion que l'accord partiel y $a$ (ev. $\left.Y A, y^{\prime} a\right)$ domine $(86 \%)$ la communication synchrone par rapport à la communication asynchrone (14\%). Cette tendance se manifeste aussi dans notre corpus, où la forme ya est prévalente.

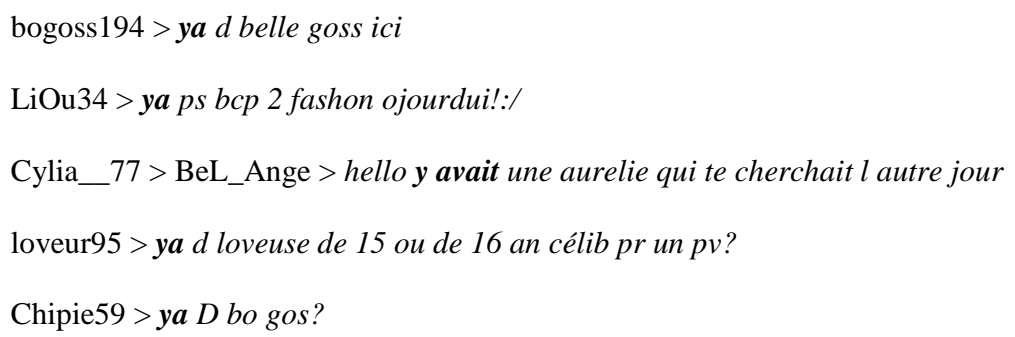

Le rapprochement du français tchaté et du français familier est observable aussi dans le cas des verbes savoir et être. Mentionnons surtout la première personne du singulier de ces verbes qu' on trouve sous la forme oralisante chui et che dans notre corpus.

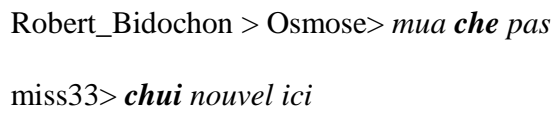

E) Le cinquième type d'accord observé dans notre corpus est un accord erroné. Nous lui attribuons cette dénomination car il ajoute au verbe des désinences inappropriées correspondant à d'autres personnes. Il nous semble intéressant d'ajouter que ce type d'accord n'est pas présent dans les SMS français. Bien que le marquage d'accord en français tchaté et en français des SMS manifeste de nombreuses ressemblances, STARK (2011 : 42) nous précise que l'accord erroné 
apparaît dans les SMS seulement en cas d'absence du contrôleur. Mentionnons quelques exemples de notre corpus :

\author{
Lililatigresse > ricardo30 > oui oui on dis ça \\ Socrate_le-boss > Jenn > il faur dessendre combin de cm pour $k$ sa te plait ????? \\ Coco44 > soso > tu fait sa esprès \\ Groslolo > vener me parlé \\ Aetaluta\{douche $\}>$ tu doit le fair??? \\ Bogosscelib > il as des personne du 62
}

D'après PANCKHURST (1998 : 33) on peut classer ce type d'erreur en deux catégories majeures : erreurs machinales/discriminantes et erreurs floues/nondiscriminantes. La première catégorie est engendrée uniquement par l'utilisation de l'ordinateur qui implique une certaine surcharge cognitive. On peut constater que ce type d'erreur n'apparaît que sur l'écran de l'ordinateur et il ne devrait pas se produire dans une forme manuscrite. Mentionnons quelques exemples de notre corpus :

$$
\begin{array}{ll}
\text { charlotte }>\text { ze zui malade } ! ! ! ! ! & \text { (je suis } \rightarrow \text { ze zui) } \\
\text { jujuM }>\text { mimi }>\text { tu ees ici } ? & \text { (tu es } \rightarrow \text { tuees) }
\end{array}
$$

Le deuxième type d'erreurs provient d'une méconnaissance des règles typographiques, orthographiques ou grammaticales et ne doit pas être obligatoirement liée au moyen de communication spécifique utilisé. Nous sommes d'accord avec PANCKHURST (1998: 34) qu'il est difficile de cerner ce type d'erreur sans profil sociologique, pourtant l'accord je n'est pas nous semble tellement surprenant et confondant que nous avons décidé de le classer comme erreur floue/non-discriminante.

filou > c normal ke sur mon home player, je n'est pas la chaine 1?

Pour conclure le paragraphe sur les différents types d'accord, nous jugeons utile de dresser un tableau récapitulatif avec leurs fréquences. 


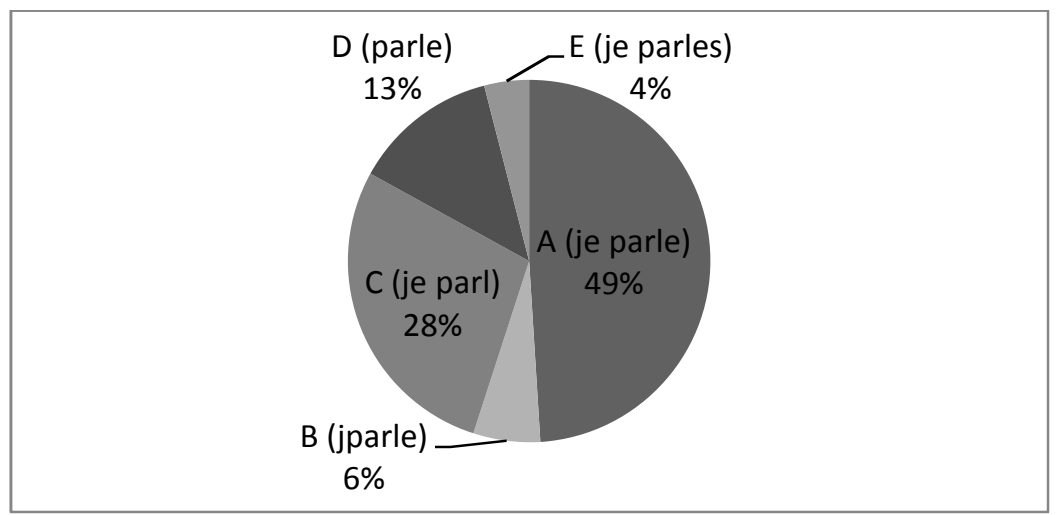

Graphique 1 : Fréquence des différents types d'accord

Le graphique ci-dessus nous montre que l'accord canonique, avec une fréquence de $49 \%$, est prépondérant par rapport à d'autres types d'accord. Il s'agit d'une constatation surprenante car nos études précédentes sur le français tchaté ont prouvé la prédominance des graphies non standard dans ce type de communication (LAZAR, 2012 : 27). Pour cette raison, nous jugeons utile de comparer la fréquence d'emploi de l'accord canonique par rapport à d'autres types d'accords qui dérogent aux règles orthographiques et grammaticales du français soutenu. Il en résulte que si on prend en considération la fréquence de tous les accords non standard, on peut constater une légère supériorité de ce type d'accord (51\%) par rapport à l'écrit standard (49\%).

\section{Conclusion}

L'étude de la morphosyntaxe du français tchaté s'est révélée être un terrain particulièrement fructueux. Quoique la problématique de l'accord verbe sujet soit évoquée dans presque chaque grammaire de la langue française, on peut constater qu'il représente un cas particulier dans le cadre de la communication médiée par ordinateur. L'expression du nombre et du genre, qui s'exprimait à travers des marques morphologiques « traditionnelles », subit sur l'écran de l'ordinateur certaines modifications. Les tchateurs jugent les marques morphologiques, qui ont évolué au cours de plusieurs siècles, comme vieillies et inutiles pour la communication tchatée et ils ressentent le besoin de les modifier et de les adapter à la rapidité de la communication tchatée. L'analyse détaillée de notre corpus nous a permis d'identifier au total 5 types d'accord dont un seul correspondait aux règles orthographiques et grammaticales du français standard. Néanmoins, il faut souligner que l'accord canonique, qui conserve le donneur et le marquage explicite et canonique de la personne et du nombre sur le verbe conjugué, se montre prévalent, avec une fréquence de $49 \%$ (c'est-à-dire de 227 items), par rapport à d'autres types d'accord. Il s'agit d'une constatation surprenante, qui a réfuté notre hypothèse de départ sur la prévalence de l'accord introduisant des marques morphologiques simplificatrices. Précisons que d'autres types d'accord, qui se sont montrés déviants par rapport au français standard, restent minoritaires. Il faut les prendre en bloc, pour pouvoir constater une légère prédominance ( 237 items) 
de ces accords par rapport à l'accord canonique (227 items). Nous en déduisons que le code graphique se montre plus résistant dans le marquage de l'accord pronom sujet-verbe conjugué que dans d'autres types d'écrit tchaté (LAZAR, 2012). Ajoutons que l'objectif de ces accords non standard est toujours le même, c'est-àdire accélérer la communication entre les individus en réduisant le nombre de caractères. Généralement, on peut constater que les tchateurs recourent à la suppression des voyelles et consonnes non prononcées, qui sont jugées redondantes. Enfin, il nous reste à signaler que les tchateurs se montrent très créatifs en proposant aux internautes de nouvelles marques morphologiques, telles que $e ́$ qui peut facilement remplacer les terminaisons du conditionnel et du futur simple ainsi que de l'indicatif et de l'impératif de certains verbes conjugués.

Pour conclure cet article, il faut se poser la question si ces nouveaux types d'accord ont de la chance d'entrer dans le système grammatical de la langue française. Rappelons que les rectifications de l'orthographe française, qui sont de plus en plus fréquentes, nous montrent que le code graphique du français pose de nombreuses difficultés non seulement aux apprenants, mais aussi aux locuteurs natifs. Il est évident que les modifications grammaticales (ou orthographiques) d'une langue n'arrivent pas d'un jour à l'autre, pourtant la prolifération de nouveaux types d'accord dans la communication médiée par ordinateur est de plus en plus évidente. Il serait sans aucun doute intéressant de comparer cette variation à travers des corpus différents, tels que Twitter ou Facebook.

\section{BIBLIOGRAPHIE}

ANIS Jacques (1998), Texte et ordinateur, l'écriture réinventée ? Bruxelles, Université de Boeck.

ANIS Jacques (1999), Internet, communication et langue française, Paris, Hermès.

ANIS Jacques (2001), Parlez-vous texto ? Paris, Le Cherche Midi.

ANIS, Jacques (2002), L'écriture, théories et descriptions, Bruxelles, Université de Boeck.

CHOVANCOVÁ Katarína (2008a), Les discussions en direct sur Internet (Énonciation et graphie), Banská Bystrica, Univerzita Mateja Bela.

CHOVAnCOVÁ Katarína (2008b), Les discussions en direct sur Internet (Aspects pragmatiques), Banská Bystrica : Univerzita Mateja Bela.

DEJOND Aurélie ; MERCIER Jacques (2002), La cyberl@ngue française, Bruxelles, La Renaissance du Livre.

GIRAUD Pierre (1974), La syntaxe du français, Paris : PUF.

GREVISSE Maurice ; GoSSE André (1991), Le Bon Usage - Grammaire française. Paris - Louvain-la-Neuve, De Boeck - Duculot.

HENDRICH Josef; RADINA Otomar; TLÁSKAL Jaromír (1992), Francouzská mluvnice, Praha, SPN.

HONOVÁ Zuzana (2008), Le rôle des procédés morphologiques pour l'expression de l'aspect en français, Studia Romanistica, č. 8, p. 99-106

JANDOVÁ Eva (2006), Čeština na WWW chatu, Ostrava, OU.

JANDOVÁ Eva (2006 b), Konverzace na WWW chatu, Ostrava, OU. 
KATZ Leonard ; FROST Ram (1992), The Reading Process is Different for Different Orthographies, in : Katz Leonard ; Frost Ram (éds), The Orthographic Depth Hypothesis, Orthography, Phonology, Morphology and Meaning, Amsterdam, Elsevier North Holland Press, p. 67-84.

LAMBERT Ludovic; KAIL Michèle (2001), Le traitement en temps réel des marques morphologiques d'accord dans des phrases françaises, L'année psychologique, vol. 101, $\mathrm{n}^{\circ}$ 4, p. 561-592.

LAZAR Jan (2012), Quelques observations sur les néographies phonétisantes en français tchaté, Linguistica pragensia, vol. XXII, $\mathrm{n}^{\circ} .1$, p.18-28

MARCOCCIA Michel (2000), La représentation du non verbal dans la communication écrite médiatisée par ordinateur, Communication et organisation

URL: http://communicationorganisation.revues.org/2431 [consulté le 10 février 2014]

PANCKHURST Rachel (1998), Marques typiques et ratages en communication médiée par Ordinateur, Actes du colloque CIDE 98, p. 31-43,

URL: $\quad$ http://recherche.univ-montp3.fr/rachel/spip/IMG/pdf/cide-c98.pdf [consulté le 10 février 2014]

PANCKHURST Rachel (1998), Analyse linguistique du courrier électronique, Actes $d u$ colloque Les relations entre individus médiatisées par les réseaux informatiques, Paris, L'Harmattan, p. 47-60.

PANCKHURST Rachel (1999), Analyse linguistique assistée par ordinateur du courriel, in : Anis J. (éd.), Internet, communication et langue française, Paris, Hermès, p. 55-70.

PANCKHURST Rachel (2006), Le discours électronique médié: bilan et perspectives, in : Piolat A. (éd.), Lire, écrire, communiquer et apprendre avec Internet, Marseille, Éditons Solal, p. 345-366.

PIEROZAK Isabelle (2000a), Les pratiques discursives des internautes en français : matériaux et éléments de réflexion, Le français moderne LXVIII, $\mathrm{n}^{\circ} 1$, p. 109129.

PIEROZAK Isabelle (2000b), Approche sociolinguistique des pratiques discursives en français sur Internet : "ge fé dais fotes si je voeux », Revue Française de Linguistique Appliquée, vol. V, fasc. 1, p. 89-104.

PIEROZAK Isabelle (2003a), «Le français tchaté » un objet à géométrie variable, Langage \& société (Écrits électroniques : échanges, usages et valeur), ${ }^{\circ}{ }^{\circ} 104$, p. 123-144.

PIEROZAK Isabelle (2003b), Contacts de langues sur internet : collisions / collusions ? L'exemple des échanges en temps réel en français », in: Billiez J.; Rispail M. (éds), Contacts de langues : modèles, typologies, interventions, Paris, L'Harmattan (coll. « Espaces discursifs »), p. 177-189.

PIEROZAK Isabelle (2005), Pour une approche syntaxique complexe : l'exemple d'objets dits «simples », La linguistique, vol. 41, fasc. 1, p. 107-131.

STARK Elisabeth (2011), La morphosyntaxe dans les SMS suisses francophones : Le marquage de l'accord sujet - verbe conjugué, Linguistik on line 48, p. 3547, URL : http://www.linguistik-online.de/48_11/stark.html [consulté le 10 février 2014] 
VAN COMPERNOLLE Rémi; WILliams Lawrence (2007), De l'oral à l'électronique : La variation orthographique comme ressource sociostylistique et pragmatique dans le français électronique, Glottopol, $\mathrm{n}^{\circ} 10$, p. 56-69, http://glottopol.univ-ouen.fr/telecharger/numero_10/gpl10_04compernolle.pdf [consulté le 10 février 2014] 